\title{
SIGMOIDAL-TYPE SERIES EXPANSION
}

\author{
BEONG IN YUN ${ }^{1}$
}

(Received 3 August, 2006; revised 10 January, 2008)

\begin{abstract}
In this paper we introduce a set of orthonormal functions, $\left\{\phi_{n}^{[r]}\right\}_{n=1}^{\infty}$, where $\phi_{n}^{[r]}$ is composed of a sine function and a sigmoidal transformation $\gamma_{r}$ of order $r>0$. Based on the proposed functions $\phi_{n}^{[r]}$ named by sigmoidal sine functions, we consider a series expansion of a function on the interval $[-1,1]$ and the related convergence analysis. Furthermore, we extend the sigmoidal transformation to the whole real line $\mathbb{R}$ and then, by reconstructing the existing sigmoidal cosine functions $\psi_{n}^{[r]}$ and the presented functions $\phi_{n}^{[r]}$, we develop two kinds of 2-periodic series expansions on $\mathbb{R}$. Superiority of the presented sigmoidal-type series in approximating a function by the partial sum is demonstrated by numerical examples.
\end{abstract}

2000 Mathematics subject classification: primary 41A58; secondary 42A20.

Keywords and phrases: Fourier series, sigmoidal transformation, sigmoidal series.

\section{Introduction}

Recently [8] the author introduced an orthonormal basis $\left\{\psi_{n}^{[r]}\right\}_{n=0}^{\infty}$, where the so-called sigmoidal cosine function $\psi_{n}^{[r]}$ is composed of a cosine function and a sigmoidal transformation $\gamma_{r}$ of order $r>0$. It was proved that a series expansion, based on $\left\{\psi_{n}^{[r]}\right\}_{n=0}^{\infty}$, of a piecewise smooth function converges on the interval $[-1,1]$. It was also shown that a partial sum of the series expansion results in a very accurate approximation to a function for which the Fourier series approximation is inadequate. However, its 2-periodic extension to the real line $\mathbb{R}$ is not complete because of the mismatch of the periods between even functions $\psi_{2 k}^{[r]}$ and odd functions $\psi_{2 k-1}^{[r]}$. This is one of the motivations of the present work. The principal results of the series based on the functions $\left\{\psi_{n}^{[r]}\right\}_{n=0}^{\infty}$ are summarized in Section 2.

The main objective of this work is to investigate several types of series expansions using the sigmoidal-type cosine and/or sine functions which can be properly extended to $\mathbb{R}$. First, following the procedure to develop the sigmoidal cosine function $\psi_{n}^{[r]}$

\footnotetext{
${ }^{1}$ School of Mathematics, Informatics and Statistics, Kunsan National University, Kunsan, 573-701, South Korea; e-mail: biyun@kunsan.ac.kr.

(C) Australian Mathematical Society 2008, Serial-fee code 0334-2700/08
} 
as given in [8], in this paper we introduce another orthonormal basis $\left\{\phi_{n}^{[r]}\right\}_{n=1}^{\infty}$ for a weighted $L_{2}$ space $L_{2, w}([-1,1])$ with respect to a weight function $w^{[r]}$ in $(2.3)$. The function $\phi_{n}^{[r]}$, named a sigmoidal sine function, is composed of a sigmoidal transformation $\gamma_{r}$ of order $r>0$ and a sine function. Concerning a series based on these sigmoidal sine functions, its convergence analysis on the interval $[-1,1]$ is given in Section 3.

Though the sigmoidal transformation $\gamma_{r}$ is fundamentally defined on a limited interval $[0,1]$, in Section 4 we suggest two types of extended sigmoidal transformations which make it possible to extend both the sigmoidal cosine and the sigmoidal sine functions to the real line $\mathbb{R}$. The periodicity of the extended sigmoidal functions is also classified.

In Section 5, using the extended sigmoidal functions, we construct two extended sigmoidal series of order $r>0$ which are 2-periodic on $\mathbb{R}$. In addition, one of these series appears to be equivalent to the Fourier series in the case of $r=1$.

Approximation of a function by the partial sum of a series is very important in practical applications. Section 6 gives numerical examples with regard to functions for which the traditional Fourier series approximation is not adequate. In the result one can observe the availability and superiority of the presented sigmoidal-type series.

\section{Preliminaries}

This work is essentially based on the sigmoidal transformation, which is a one-toone mapping of the interval $[0,1]$ onto itself taking the shape of an elongated ' $\mathrm{S}$ '. It is well known that coordinate transformation techniques combined with the sigmoidal transformation are very efficient for accurate numerical evaluation of singular integrals [1-5, 7, 9-11].

For application to a series expansion, we recall the sigmoidal transformation as follows.

DEFINITION 1. For any $r>0$ a real-valued function, denoted by $\gamma_{r}(y)$, which has the following properties is called a sigmoidal transformation of order $r$ :

(i) $\gamma_{r}(y) \in \mathrm{C}[0,1] \cap \mathrm{C}^{\infty}(0,1)$;

(ii) $\gamma_{r}(y)+\gamma_{r}(1-y)=1,0 \leq y \leq 1$;

(iii) $\gamma_{r}(y)$ is strictly increasing on $[0,1]$ with $\gamma_{r}(0)=0$;

(iv) on the subinterval $[0,1 / 2] \gamma_{r}^{\prime}(y)$ is strictly increasing when $r>1$, and it is strictly decreasing when $r<1$; and

(v) near $y=0, \gamma_{r}^{(j)}(y)=O\left(y^{r-j}\right)$ for $j=0,1,2, \ldots,\lfloor r\rfloor$, where $\lfloor r\rfloor$ denotes the greatest integer less than or equal to $r$.

Particularly, for $r=1$, it is assumed that $\gamma_{1}(y):=y$.

Among several existing sigmoidal transformations we will use the simplest one [6] in the form of

$$
\gamma_{r}(y)=\frac{y^{r}}{y^{r}+(1-y)^{r}}, \quad 0 \leq y \leq 1,
$$

through the present work. We note that its inverse is $\gamma_{r}^{-1}=\gamma_{1 / r}$. 
Recently [8] the author presented a so-called sigmoidal cosine function of order $r>0$ such as

$$
\psi_{k}^{[r]}(x):=\cos \left[k \pi \gamma_{r}\left(\frac{1+x}{2}\right)\right], \quad k=1,2,3, \ldots,
$$

with $\psi_{0}^{[r]}(x):=1 / \sqrt{2},-1 \leq x \leq 1$. For a weight function

$$
w^{[r]}(x):=\gamma_{r}^{\prime}\left(\frac{1+x}{2}\right)
$$

and an inner product of functions $f$ and $g$ defined by

$$
\langle f, g\rangle_{w}:=\int_{-1}^{1} w^{[r]}(x) f(x) g(x) d x,
$$

the set $\left\{\psi_{k}^{[r]}\right\}_{k=0}^{\infty}$ is an orthonormal basis for the weighted $L_{2}$ space, $L_{2, w}([-1,1])$ equipped with a norm $\|f\|_{2, w}=\langle f, f\rangle_{w}^{1 / 2}$. The author has presented a series expansion of a function $f$ as follows:

$$
\begin{aligned}
S_{\cos }^{[r]}(f ; x) & =\sum_{n=0}^{\infty} c_{n} \psi_{n}^{[r]}(x) \\
& =\frac{c_{0}}{\sqrt{2}}+\sum_{n=1}^{\infty} c_{n} \cos \left[\pi n \gamma_{r}\left(\frac{1+x}{2}\right)\right], \quad-1 \leq x \leq 1,
\end{aligned}
$$

where the coefficient $c_{n}$ is

$$
c_{n}=\int_{-1}^{1} w^{[r]}(t) \psi_{n}^{[r]}(t) f(t) d t
$$

We call the series $S_{\mathrm{cos}}^{[r]}(f ; x)$ a sigmoidal cosine series (SM-CS) of $f(x)$ of order $r>0$.

Now we summarize the main results of the sigmoidal cosine functions $\psi_{k}^{[r]}$ and the SM-CS $S_{\cos }^{[r]}(f ; x)$ which were proved in [8].

THEOREM 2.1. For each integer $k \geq 1$ the function $\psi_{k}^{[r]}(x),-1 \leq x \leq 1$, has the following properties:

(1) $\psi_{k}^{[r]}(-1)=1, \psi_{k}^{[r]}(0)=\cos [k \pi / 2], \psi_{k}^{[r]}(1)=(-1)^{k}$;

(2) $\psi_{k}^{[r]}(-x)=(-1)^{k} \psi_{k}^{[r]}(x)$; 
(3) near the end-points $x=-1$ and $x=1$, respectively,

$$
\psi_{k}^{[r]}(x)=1+O\left((1+x)^{2 r}\right), \quad \psi_{k}^{[r]}(x)=(-1)^{k}+O\left((1-x)^{2 r}\right)
$$

(4) the zeros of $\psi_{k}^{[r]}$ are

$$
x_{k, j}^{[r]}=2 \gamma_{r}^{-1}\left(\frac{2 j-1}{2 k}\right)-1, \quad j=1,2, \ldots, k .
$$

We recall that a function $f$ is called piecewise continuous on an interval $[a, b]$ if it has at most a finite number of points of discontinuity, and, in addition, the onesided limits exist at each point of discontinuity on the interval. Furthermore, if the first derivative $f^{\prime}$ is also piecewise continuous, $f$ is said to be piecewise smooth. Additionally, we denote by $f\left(x_{-}\right)$and $f\left(x_{+}\right)$the left- and right-side limits of $f(x)$, respectively.

THEOREM 2.2. Let $f$ be a piecewise smooth function on an interval $[-1,1]$. Then for any $r>0$ and for each $-1<x<1$, the Nth partial sum $S_{\cos , N}^{[r]}(f ; x)$ of $S_{\cos }^{[r]}(f ; x)$ converges such that

$$
\lim _{N \rightarrow \infty} S_{\cos , N}^{[r]}(f ; x)=\frac{1}{2}\left\{f\left(x_{-}\right)+f\left(x_{+}\right)\right\}
$$

and, at the end-points $x= \pm 1$, for $0<r \leq 1$,

$$
\lim _{N \rightarrow \infty} S_{\cos , N}^{[r]}(f ; 1)=f\left(1_{-}\right), \quad \lim _{N \rightarrow \infty} S_{\cos , N}^{[r]}(f ;-1)=f\left(-1_{+}\right),
$$

respectively.

Additionally, if $f$ is continuous on $[-1,1]$ then for $0<r \leq 1$ the series $S_{\mathrm{cos}}^{[r]}(f ; x)$ converges uniformly to $f(x)$ on $[-1,1]$.

THEOREM 2.3. Let $f$ be a piecewise smooth function on any interval $[a, b]$ such that $-1<a<b<1$ and assume that near $x=1$ and $x=-1$ the behaviour of $f$ is, respectively,

$$
f(x) \sim A_{1}+B_{1}(1-x)^{\eta_{1}}, \quad f(x) \sim A_{2}+B_{2}(1+x)^{\eta_{2}},
$$

where $\eta_{1}, \eta_{2}>0$, and $A_{i}$ and $B_{i}$ are constants with $B_{1}^{2}+B_{2}^{2} \neq 0$. Then for any $r$ such that $0<r \leq \eta=\min \left\{\eta_{1}, \eta_{2}\right\}$ we have the following results.

(1) The partial sum $S_{\cos , N}^{[r]}(f ; x)$ converges on the interval $[-1,1]$ in the form of (2.7) and (2.8).

(2) If, in addition, $f$ is continuous on $[-1,1]$ then the series $S_{\mathrm{cos}}^{[r]}(f ; x)$ converges uniformly to $f(x)$ on $[-1,1]$. 


\section{A sigmoidal sine series}

As a counterpart of the function $\psi_{k}^{[r]}$ in (2.2) we define

$$
\phi_{k}^{[r]}(x):=\sin \left[k \pi \gamma_{r}\left(\frac{1+x}{2}\right)\right], \quad k=1,2,3, \ldots,
$$

on $[-1,1]$, which is called a sigmoidal sine function of order $r>0$. For a set of sigmoidal sine functions $\left\{\phi_{k}^{[r]}\right\}_{k=1}^{\infty}$, by a change of variables such as $t=\gamma_{r}((1+x) / 2)$, we can see its orthonormality as follows:

$$
\begin{aligned}
\left\langle\phi_{i}^{[r]}, \phi_{j}^{[r]}\right\rangle_{w} & :=\int_{-1}^{1} w^{[r]}(x) \phi_{i}^{[r]}(x) \phi_{j}^{[r]}(x) d x \\
& =2 \int_{0}^{1} \sin (i \pi t) \sin (j \pi t) d t=\delta_{i j},
\end{aligned}
$$

for all $i, j \geq 1$. Furthermore, not only the sigmoidal cosine functions $\left\{\psi_{k}^{[r]}\right\}_{k=0}^{\infty}$ but also the sigmoidal sine functions $\left\{\phi_{k}^{[r]}\right\}_{k=1}^{\infty}$ form an orthonormal basis for the weighted $L_{2}$ space, $L_{2, w}([-1,1])$, with respect to the weight function $w^{[r]}$ in $(2.3)$. The other main properties of $\phi_{k}^{[r]}$ are detailed in the following theorem. The proof is omitted since it is clear from the definition of $\phi_{k}^{[r]}$ in (3.1) and the properties of the sigmoidal transformation $\gamma_{r}$.

THEOREM 3.1. For each integer $k \geq 1$ the function $\phi_{k}^{[r]}(x),-1 \leq x \leq 1$, has the following properties:

(1) $\phi_{k}^{[r]}(-1)=\phi_{k}^{[r]}(1)=0, \phi_{k}^{[r]}(0)=\sin [k \pi / 2]$;

(2) $\phi_{k}^{[r]}(-x)=(-1)^{k+1} \phi_{k}^{[r]}(x)$;

(3) near $x=-1$ and $x=1$,

$$
\phi_{k}^{[r]}(x)=O\left(\left(1-x^{2}\right)^{r}\right)
$$

(4) the zeros of $\phi_{k}^{[r]}$ are

$$
\xi_{k, j}^{[r]}=2 \gamma_{r}^{-1}\left(\frac{j}{k}\right)-1, \quad j=0,1,2, \ldots, k .
$$

For a piecewise continuous function $f$ on an interval $[-1,1]$ we consider a series, named a sigmoidal sine series (SM-SS) of order $r>0$, such that

$$
\begin{aligned}
S_{\sin }^{[r]}(f ; x) & :=\sum_{n=1}^{\infty} d_{n} \phi_{n}^{[r]}(x) \\
& =\sum_{n=1}^{\infty} d_{n} \sin \left[\pi n \gamma_{r}\left(\frac{1+x}{2}\right)\right], \quad-1 \leq x \leq 1,
\end{aligned}
$$


where the coefficient $d_{n}$ is

$$
d_{n}=\int_{-1}^{1} w^{[r]}(t) \phi_{n}^{[r]}(t) f(t) d t .
$$

By changing variables as $s=\gamma_{r}(1+t / 2)$, the coefficients in (3.4) can be represented by

$$
d_{n}=2 \int_{0}^{1} \sin [\pi n s] f\left(2 \gamma_{r}^{-1}(s)-1\right) d s .
$$

Referring to Theorems 2.2 and 2.3 and the related convergence analysis for the SM-CS $S_{\mathrm{cos}}^{[r]}$ in [8], we can obtain similar results for the SM-SS $S_{\sin }^{[r]}$ as well. These are included in the following theorems, whose proofs are omitted as they are parallel to the case of the SM-CS.

THEOREM 3.2. Let $f$ be a piecewise smooth function on an interval $[-1,1]$. Then for any $r>0$ and for each $-1<x<1$, the N th partial sum $S_{\sin , N}^{[r]}(f ; x)$ of $S_{\sin }^{[r]}(f ; x)$ converges such that

$$
\lim _{N \rightarrow \infty} S_{\sin , N}^{[r]}(f ; x)=\frac{1}{2}\left\{f\left(x_{-}\right)+f\left(x_{+}\right)\right\},
$$

and, at the end-points $x= \pm 1$, for $0<r \leq 1$,

$$
\lim _{N \rightarrow \infty} S_{\sin , N}^{[r]}(f ; 1)=\lim _{N \rightarrow \infty} S_{\sin , N}^{[r]}(f ;-1)=0 .
$$

Additionally, if $f$ is continuous on $[-1,1]$ and $f(-1)=f(1)=0$ then for $0<r \leq 1$ the series $S_{\sin }^{[r]}(f ; x)$ converges uniformly to $f(x)$ on $[-1,1]$.

THEOREM 3.3. Let $f$ satisfy all the conditions in Theorem 2.3. Then for $0<r \leq \eta$ $=\min \left\{\eta_{1}, \eta_{2}\right\}$ we have the following results.

(1) The partial sum $S_{\text {sin, } N}^{[r]}(f ; x)$ converges on the interval $[-1,1]$ in the form of (3.6) and (3.7).

(2) If, in addition, $f$ is continuous on $[-1,1]$ and $f(-1)=f(1)=0$ then the series $S_{\sin }^{[r]}(f ; x)$ converges uniformly to $f(x)$ on $[-1,1]$.

The condition $f(-1)=f(1)=0$ in Theorems 3.2 and 3.3(2) can be weakened, such as $f(-1)=f(1)$, because, in this case, one has only to add a constant $f(1)$ to the series $S_{\sin }^{[r]}(F ; x)$ of the redefined function $F(x)=f(x)-f(1)$.

\section{Extension of the sigmoidal transformation to $\mathbb{R}$}

The sigmoidal transformation $\gamma_{r}$ is defined on a finite interval $[0,1]$ fundamentally, and thus the sigmoidal sine or cosine series in itself cannot be used for a function defined on the real line $\mathbb{R}$. Fortunately, however, we can make it possible by some proper modifications. 
We consider an extension of the sigmoidal transformation $\gamma_{r}$ onto $\mathbb{R}$ such as

$$
\gamma_{r}^{*}(x):=\gamma_{r}\left(\left|x-2\left\lfloor\frac{x+1}{2}\right\rfloor\right|\right), \quad x \in \mathbb{R} .
$$

Since $\left|x-2\left\lfloor\frac{1}{2}(x+1)\right\rfloor\right| \leq 1$ for every real number $x$, the function $\gamma_{r}^{*}(x)$ is well defined.

LEMMA 4.1. For all $x \in \mathbb{R}$, it follows that:

(1) $\gamma_{r}^{*}(x)$ is continuous;

(2) $\gamma_{r}^{*}(x)$ is 2-periodic and even; and

(3) $\gamma_{r}^{*}(x)+\gamma_{r}^{*}(1-x)=1$.

PROOF. For the property (1) we only have to check the continuity at any integer $k$. The left- and right-side limits respectively become

$$
\begin{aligned}
\lim _{x \rightarrow k-} \gamma_{r}^{*}(x) & \left.=\lim _{x \rightarrow k-} \gamma_{r}\left(|x-2| \frac{x+1}{2}\right\rfloor \mid\right) \\
& = \begin{cases}\gamma_{r}\left(\left|k-2\left(\frac{k-1}{2}\right)\right|\right)=\gamma_{r}(1)=1 & (k: \text { odd }) \\
\gamma_{r}\left(\left|k-2\left(\frac{k}{2}\right)\right|\right)=\gamma_{r}(0)=0 & (k: \text { even }),\end{cases}
\end{aligned}
$$

and

$$
\lim _{x \rightarrow k+} \gamma_{r}^{*}(x)= \begin{cases}\gamma_{r}\left(\left|k-2\left(\frac{k+1}{2}\right)\right|\right)=\gamma_{r}(1)=1 & (k: \text { odd }), \\ \gamma_{r}\left(\left|k-2\left(\frac{k}{2}\right)\right|\right)=\gamma_{r}(0)=0 & (k: \text { even }),\end{cases}
$$

which implies that $\gamma_{r}^{*}(x)$ is continuous at $x=k$.

For any integer $x=k$ the property (2) holds from the results above. Moreover, noting that

$$
\lfloor\xi+1\rfloor=\lfloor\xi\rfloor+1, \quad\lfloor-\xi\rfloor=-\lfloor\xi\rfloor-1,
$$

for a noninteger $\xi$, we can prove the property (2) for any noninteger $x$ as follows:

$$
\begin{aligned}
\gamma_{r}^{*}(x+2) & =\gamma_{r}\left(\left|x+2-2\left\lfloor\frac{x+1}{2}+1\right\rfloor\right|\right) \\
& =\gamma_{r}\left(\left|x+2-2\left\lfloor\frac{x+1}{2}\right\rfloor-2\right|\right) \\
& =\gamma_{r}\left(\left|x-2\left\lfloor\frac{x+1}{2}\right\rfloor\right|\right)=\gamma_{r}^{*}(x)
\end{aligned}
$$


and

$$
\begin{aligned}
\gamma_{r}^{*}(-x) & =\gamma_{r}\left(\left|-x-2\left\lfloor\frac{-x+1}{2}\right\rfloor\right|\right) \\
& =\gamma_{r}\left(\left|x+2\left\lfloor\frac{-x+1}{2}\right\rfloor\right|\right) \\
& =\gamma_{r}\left(\left|x-2\left(\left\lfloor\frac{x-1}{2}\right\rfloor+1\right)\right|\right) \\
& =\gamma_{r}\left(\left|x-2\left\lfloor\frac{x+1}{2}\right\rfloor\right|\right)=\gamma_{r}^{*}(x) .
\end{aligned}
$$

For the property (3) we observe that

$$
\gamma_{r}^{*}(x)=\gamma_{r}\left(\left\lfloor x-2\left\lfloor\frac{x+1}{2}\right\rfloor \mid\right), \quad \gamma_{r}^{*}(1-x)=\gamma_{r}\left(\left|1-x-2\left\lfloor\frac{-x+2}{2}\right\rfloor\right|\right) .\right.
$$

For $0<x<1$, since $\left\lfloor\frac{1}{2}(x+1)\right\rfloor=\left\lfloor\frac{1}{2}(-x+2)\right\rfloor=0$, we have

$$
\gamma_{r}^{*}(x)+\gamma_{r}^{*}(1-x)=\gamma_{r}(x)+\gamma_{r}(1-x)=1 .
$$

For $1 \leq x \leq 2$, since $\left\lfloor\frac{1}{2}(x+1)\right\rfloor=1$ and $\left\lfloor\frac{1}{2}(-x+2)\right\rfloor=0$, we have

$$
\begin{aligned}
\gamma_{r}^{*}(x)+\gamma_{r}^{*}(1-x) & =\gamma_{r}(|x-2|)+\gamma_{r}(|1-x|) \\
& =\gamma_{r}(1-(x-1))+\gamma_{r}(x-1)=1 .
\end{aligned}
$$

Noting that $\gamma_{r}^{*}$ is a 2-periodic even function as shown previously, one can see that the equation in (3) is valid for all $x \in \mathbb{R}$.

For an integer $k$ we denote by $\psi_{k}^{*[r]}$ and $\phi_{k}^{*[r]}$ the sigmoidal cosine and sine functions defined as

$$
\psi_{k}^{*[r]}(x):=\cos \left[k \pi \gamma_{r}^{*}\left(\frac{1+x}{2}\right)\right], \quad \phi_{k}^{*[r]}(x):=\sin \left[k \pi \gamma_{r}^{*}\left(\frac{1+x}{2}\right)\right],
$$

for all $x \in \mathbb{R}$. Then, from Lemma 4.1, it follows that

$$
\begin{aligned}
\psi_{k}^{*[r]}(x+2) & =\cos \left[k \pi \gamma_{r}^{*}\left(1+\frac{1+x}{2}\right)\right] \\
& =\cos \left[k \pi \gamma_{r}^{*}\left(1-\frac{1+x}{2}\right)\right] \\
& =\cos \left[k \pi\left\{1-\gamma_{r}^{*}\left(\frac{1+x}{2}\right)\right\}\right]=(-1)^{k} \psi_{k}^{*[r]}(x),
\end{aligned}
$$

and similarly

$$
\phi_{k}^{*[r]}(x+2)=\sin \left[k \pi\left\{1-\gamma_{r}^{*}\left(\frac{1+x}{2}\right)\right\}\right]=(-1)^{k+1} \phi_{k}^{*[r]}(x) .
$$




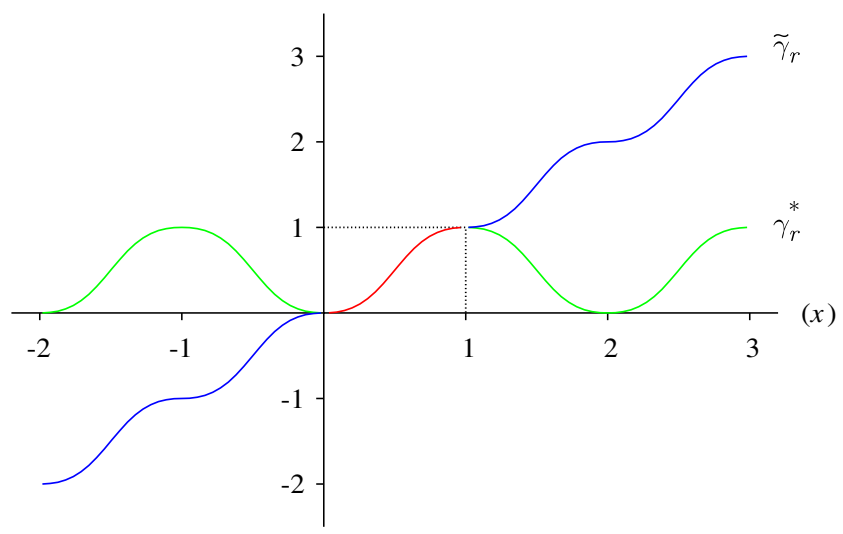

FIGURE 1. Extended sigmoidal transformations $\gamma_{r}^{*}(x)$ and $\tilde{\gamma}_{r}(x),-2 \leq x \leq 3$, with $r=2$.

Therefore the even functions $\psi_{2 n}^{*[r]}$ and $\phi_{2 n-1}^{*[r]}$ are 2-periodic while the odd functions $\psi_{2 n-1}^{*[r]}$ and $\phi_{2 n}^{*[r]}$ are 4-periodic. This mismatch of the periods between the even and odd sigmoidal functions is not reasonable in approximation to arbitrary 2-periodic functions on $\mathbb{R}$ in general.

As an alternative idea we suggest another extension of $\gamma_{r}$ such as

$$
\tilde{\gamma}_{r}(x):=\lfloor x\rfloor+\gamma_{r}(x-\lfloor x\rfloor), \quad x \in \mathbb{R} .
$$

Although $\tilde{\gamma}_{r}(x)$ is not periodic in $x$, for any integer $k$ its graphs on every interval $[k, k+1]$ are repetitive and they are glued continuously at the end-points $x=k$ and $x=k+1$. Rigorous investigation for these properties is given in the following lemma and the global behaviour of $\widetilde{\gamma}_{r}(x)$ is shown in Figure 1 in comparison with $\gamma_{r}^{*}(x)$.

LEMMA 4.2. For all $x \in \mathbb{R}$, it follows that:

(1) $\tilde{\gamma}_{r}(x)$ is continuous;

(2) $\tilde{\gamma}_{r}(1+x)=1+\tilde{\gamma}_{r}(x)$; and

(3) $\widetilde{\gamma}_{r}(-x)=-\widetilde{\gamma}_{r}(x)$, that is, $\tilde{\gamma}_{r}$ is an odd function.

PROOF. For the property (1) it is sufficient to see the continuity at $x=k$ for each integer $k$. That is,

$$
\lim _{x \rightarrow k-} \tilde{\gamma}_{r}(x)=k-1+\lim _{x \rightarrow k-} \gamma_{r}(x-(k-1))=k-1+\gamma_{r}(1)=k
$$

and

$$
\lim _{x \rightarrow k+} \tilde{\gamma}_{r}(x)=k+\lim _{x \rightarrow k+} \gamma_{r}(x-k)=k+\gamma_{r}(0)=k .
$$

The property (2) results from

$$
\begin{aligned}
\tilde{\gamma}_{r}(1+x) & =\lfloor 1+x\rfloor+\gamma_{r}(1+x-\lfloor 1+x\rfloor) \\
& =1+\lfloor x\rfloor+\gamma_{r}(x-\lfloor x\rfloor)=1+\widetilde{\gamma}_{r}(x) .
\end{aligned}
$$


Finally, for each integer $x$ it can be seen that $\tilde{\gamma}_{r}(-x)=-x+\gamma_{r}(0)=-\widetilde{\gamma}_{r}(x)$ clearly. On the other hand let $x$ be noninteger. Then since $\lfloor-x\rfloor=-\lfloor x\rfloor-1$ it follows that

$$
\begin{aligned}
\tilde{\gamma}_{r}(-x) & =-\lfloor x\rfloor-1+\gamma_{r}(-x+\lfloor x\rfloor+1) \\
& =-\lfloor x\rfloor-1+1-\gamma_{r}(x-\lfloor x\rfloor) \\
& =-\lfloor x\rfloor-\gamma_{r}(x-\lfloor x\rfloor)=-\widetilde{\gamma}_{r}(x) .
\end{aligned}
$$

The second equality results from the fact that $\gamma_{r}(1-\alpha)=1-\gamma_{r}(\alpha)$ for $0 \leq \alpha \leq 1$ as given in Definition 1(ii). Thus the property (3) has been proved.

We now denote the sigmoidal cosine and sine functions combined with $\tilde{\gamma}_{r}$ by $\widetilde{\psi}_{k}^{[r]}$ and $\widetilde{\phi}_{k}^{[r]}$, respectively. That is, for any integer $k$,

$$
\widetilde{\psi}_{k}^{[r]}(x):=\cos \left[k \pi \widetilde{\gamma}_{r}\left(\frac{1+x}{2}\right)\right], \quad \widetilde{\phi}_{k}^{[r]}(x):=\sin \left[k \pi{\widetilde{\gamma_{r}}}^{\left.\left(\frac{1+x}{2}\right)\right],}\right.
$$

with $x \in \mathbb{R}$. Naturally $\widetilde{\psi}_{k}^{[r]}$ and $\widetilde{\phi}_{k}^{[r]}$ are, respectively, extensions of $\psi_{k}^{[r]}$ and $\phi_{k}^{[r]}$ since

$$
\widetilde{\psi}_{k}^{[r]}(x)=\psi_{k}^{[r]}(x), \quad \widetilde{\phi}_{k}^{[r]}(x)=\phi_{k}^{[r]}(x),
$$

on the interval $[-1,1]$.

LEMMA 4.3. For any integer $n \geq 1$ both the even sigmoidal cosine function $\widetilde{\psi}_{2 n}^{[r]}$ and the odd sigmoidal sine function $\widetilde{\phi}_{2 n}^{[r]}$ are 2-periodic on $\mathbb{R}$. In addition, $\widetilde{\psi}_{2 n-1}^{[r]}$ and $\widetilde{\phi}_{2 n-1}^{[r]}$ are 4-periodic.

Proof. From Lemma 4.2(2) we have, for any integer $k$,

$$
\begin{aligned}
\widetilde{\psi}_{k}^{[r]}(x+2) & =\cos \left[k \pi \widetilde{\gamma}_{r}\left(1+\frac{1+x}{2}\right)\right] \\
& =\cos \left[k \pi\left\{1+\tilde{\gamma}_{r}\left(\frac{1+x}{2}\right)\right\}\right]=(-1)^{k} \widetilde{\psi}_{k}^{[r]}(x),
\end{aligned}
$$

and

$$
\widetilde{\phi}_{k}^{[r]}(x+2)=\sin \left[k \pi\left\{1+\widetilde{\gamma}_{r}\left(\frac{1+x}{2}\right)\right\}\right]=(-1)^{k} \widetilde{\phi}_{k}^{[r]}(x) .
$$

This implies that both $\widetilde{\psi}_{2 n}^{[r]}$ and $\widetilde{\phi}_{2 n}^{[r]}$ are 2-periodic. Additionally, it follows straightforwardly that $\widetilde{\psi}_{2 n-1}^{[r]}(x+4)=\widetilde{\psi}_{2 n-1}^{[r]}(x)$ and $\widetilde{\phi}_{2 n-1}^{[r]}(x+4)=\widetilde{\phi}_{2 n-1}^{[r]}(x)$.

In Table 1 the extended sigmoidal functions discussed above are classified according to their properties. The possible choices of the suitable 2-periodic orthonormal basis, with respect to the interval $[-1,1]$, are as follows:

(B1) $\left\{\widetilde{\psi}_{2 n}^{[r]}\right\}_{n=0}^{\infty} \cup\left\{\widetilde{\phi}_{2 n}^{[r]}\right\}_{n=1}^{\infty}$;

(B2) $\left\{\phi_{2 n-1}^{*[r]}\right\}_{n=1}^{\infty} \cup\left\{\widetilde{\phi}_{2 n}^{[r]}\right\}_{n=1}^{\infty}$. 
TABLE 1. Classification of the extended sigmoidal functions.

\begin{tabular}{lcc}
\hline & 2-periodic & 4-periodic \\
\hline Even & $\widetilde{\psi}_{2 n}^{[r]}=\psi_{2 n}^{*[r]}$ & $\widetilde{\phi}_{2 n-1}^{[r]}$ \\
functions & $\phi_{2 n-1}^{*[r]}$ & \\
Odd & $\widetilde{\phi}_{2 n}^{[r]}$ & $\widetilde{\psi}_{2 n-1}^{[r]}=\psi_{2 n-1}^{*[r]}$ \\
functions & & $\phi_{2 n}^{*[r]}$ \\
\hline
\end{tabular}

\section{Extended sigmoidal series on $\mathbb{R}$}

Now, for a function $f$ assumed to be 2-periodic on $\mathbb{R}$, a desirable 2-periodic extension of the sigmoidal cosine/sine series to the whole real line can be realized by employing the extended sigmoidal functions developed in the previous section. Taking account of the basis (B1), we define an extended sigmoidal series (ESM-S) of order $r>0$ in the form

$$
\widetilde{S}^{[r]}(f ; x):=\sum_{n=0}^{\infty} c_{2 n} \widetilde{\psi}_{2 n}^{[r]}(x)+\sum_{n=1}^{\infty} d_{2 n} \widetilde{\phi}_{2 n}^{[r]}(x),
$$

which is 2-periodic on $\mathbb{R}$, and the coefficients are

$$
c_{2 n}=\int_{-1}^{1} w^{[r]}(t) \psi_{2 n}^{[r]}(t) f(t) d t, \quad d_{2 n}=\int_{-1}^{1} w^{[r]}(t) \phi_{2 n}^{[r]}(t) f(t) d t .
$$

In particular, when $f=f_{\text {even }}$ is an even function,

$$
c_{2 n}=2 \int_{0}^{1} w^{[r]}(t) \psi_{2 n}^{[r]}(t) f_{\mathrm{even}}(t) d t, \quad d_{2 n}=0,
$$

and when $f=f_{\text {odd }}$ is an odd function,

$$
c_{2 n}=0, \quad d_{2 n}=2 \int_{0}^{1} w^{[r]}(t) \phi_{2 n}^{[r]}(t) f_{\text {odd }}(t) d t .
$$

It is worthwhile to note the case of $r=1$. Since $\tilde{\gamma}_{1}(x)=x$,

$$
\widetilde{\psi}_{2 n}^{[1]}(x)=(-1)^{n} \cos (n \pi x), \quad \widetilde{\phi}_{2 n}^{[1]}(x)=(-1)^{n} \sin (n \pi x),
$$

with $w^{[1]} \equiv 1$. Therefore, from (5.1) and (5.2), it follows that

$$
\widetilde{S}^{[1]}(f ; x)=\frac{a_{0}}{2}+\sum_{n=1}^{\infty} a_{n} \cos (n \pi x)+\sum_{n=1}^{\infty} b_{n} \sin (n \pi x),
$$


where $a_{n}$ and $b_{n}$ are just the Fourier coefficients. This implies that the presented series $\widetilde{S}^{[r]}(f ; x)$ in the form of $(5.1)$ is a generalization of the Fourier series according to an auxiliary parameter $r>0$ preserving its periodicity. In order to approximate a 2-periodic function $f$ on $\mathbb{R}$ by a partial sum of the series, we define the $N$ th partial sum of the extended sigmoidal series $\widetilde{S}^{[r]}(f ; x)$ as

$$
\widetilde{S}_{N}^{[r]}(f ; x):=\sum_{n=0}^{N} c_{2 n} \widetilde{\psi}_{2 n}^{[r]}(x)+\sum_{n=1}^{N} d_{2 n} \widetilde{\phi}_{2 n}^{[r]}(x) .
$$

For a 2-periodic function $f$ such that $f(-1)=f(1)=0$, by using the basis (B2) given in the previous section, one can also consider another series of the form

$$
\widetilde{S}_{\sin }^{*[r]}(f ; x):=\sum_{n=1}^{\infty} d_{2 n-1} \phi_{2 n-1}^{*[r]}(x)+\sum_{n=1}^{\infty} d_{2 n} \widetilde{\phi}_{2 n}^{[r]}(x),
$$

where the coefficients are

$$
d_{k}=\int_{-1}^{1} w^{[r]}(t) \phi_{k}^{[r]}(t) f(t) d t
$$

for all integers $k \geq 1$. The series $\widetilde{S}_{\sin }^{*[r]}$ is called an extended sigmoidal sine series (ESM-SS) of order $r>0$. One can note that $\widetilde{S}_{\sin }^{*[r]}=S_{\text {sin }}^{[r]}$ on the interval $[-1,1]$, that is, $\widetilde{S}_{\sin }^{*[r]}$ is a 2-periodic extension of $S_{\text {sin }}^{[r]}$ to the real line $\mathbb{R}$.

On the other hand, for a function $f$ defined only on the interval $[-1,1]$ where $\widetilde{\psi}_{2 n}^{[r]}=\psi_{2 n}^{[r]}$ and $\widetilde{\phi}_{2 n}^{[r]}=\phi_{2 n}^{[r]}$, we denote its series expansion in (5.1) as

$$
S^{[r]}(f ; x):=\sum_{n=0}^{\infty} c_{2 n} \psi_{2 n}^{[r]}(x)+\sum_{n=1}^{\infty} d_{2 n} \phi_{2 n}^{[r]}(x), \quad-1 \leq x \leq 1,
$$

which is called a sigmoidal series (SM-S). We recall that in this case both the SM-CS $S_{\mathrm{cos}}^{[r]}(f ; x)=\sum_{n=0}^{\infty} c_{n} \psi_{n}^{[r]}(x)$ in $(2.5)$ and the SM-SS $S_{\sin }^{[r]}(f ; x)=\sum_{n=1}^{\infty} d_{n} \phi_{n}^{[r]}(x)$ in (3.3) are also available because the periodicity does not need to be considered. Finally, we have the following theorem regarding the convergence of the SM-S $S^{[r]}$ on the interval $[-1,1]$.

THEOREM 5.1. Let $f$ be a piecewise smooth function on an interval $[-1,1]$. Then for each $-1<x<1$, the N th partial sum $S_{N}^{[r]}$ of the SM-S $S^{[r]}$ converges such that

$$
\lim _{N \rightarrow \infty} S_{N}^{[r]}(f ; x)=\frac{1}{2}\left\{f\left(x_{-}\right)+f\left(x_{+}\right)\right\}
$$

for any $r>0$ and, at both end-points $x= \pm 1$, it converges to

$$
\frac{1}{2}\left(f\left(1_{-}\right)+f\left(-1_{+}\right)\right)
$$

for $0<r \leq 1$.

Additionally, if $f$ is continuous on $[-1,1]$ and $f(-1)=f(1)$ then the SM-S $S^{[r]}$ with $0<r \leq 1$ converges uniformly to $f(x)$ on $[-1,1]$. 
PROOF. Set

$$
\gamma_{r}\left(\frac{1+x}{2}\right)=\frac{1+\xi}{2}, \quad-1 \leq \xi \leq 1,
$$

then in the formula (5.7)

$$
\begin{aligned}
& \psi_{2 n}^{[r]}(x)=\cos \left[2 n \pi \gamma_{r}\left(\frac{1+x}{2}\right)\right]=\cos [n \pi(1+\xi)]=(-1)^{n} \cos (n \pi \xi), \\
& \phi_{2 n}^{[r]}(x)=\sin \left[2 n \pi \gamma_{r}\left(\frac{1+x}{2}\right)\right]=\sin [n \pi(1+\xi)]=(-1)^{n} \sin (n \pi \xi),
\end{aligned}
$$

and the coefficients become

$$
\begin{aligned}
& c_{2 n}=(-1)^{n} \int_{-1}^{1} \cos (n \pi s) f\left(2 \gamma_{r}^{-1}\left(\frac{1+s}{2}\right)-1\right) d s, \\
& d_{2 n}=(-1)^{n} \int_{-1}^{1} \sin (n \pi s) f\left(2 \gamma_{r}^{-1}\left(\frac{1+s}{2}\right)-1\right) d s .
\end{aligned}
$$

We define a function $h$ as

$$
h(\xi)=f\left(2 \gamma_{r}^{-1}\left(\frac{1+\xi}{2}\right)-1\right),
$$

which is piecewise smooth on any subinterval $[a, b]$ of $[-1,1]$ with $-1<a<b<1$. Therefore, if we denote by $S_{N}^{\mathrm{F}}$ the $N$ th partial sum of the Fourier series then it follows that for $-1<x<1$ (that is, $-1<\xi<1$ )

$$
\begin{aligned}
\lim _{N \rightarrow \infty} S_{N}^{[r]}(f ; x) & =\lim _{N \rightarrow \infty} S_{N}^{\mathrm{F}}(h ; \xi) \\
& =\frac{1}{2}\left\{h\left(\xi_{-}\right)+h\left(\xi_{+}\right)\right\} \\
& =\frac{1}{2}\left\{f\left(2 \gamma_{r}^{-1}\left(\frac{1+\xi_{-}}{2}\right)-1\right)+f\left(2 \gamma_{r}^{-1}\left(\frac{1+\xi_{+}}{2}\right)-1\right)\right\} \\
& =\frac{1}{2}\left\{f\left(x_{-}\right)+f\left(x_{+}\right)\right\},
\end{aligned}
$$

for any $r>0$. The last equality holds from the fact that $\gamma_{r}^{-1}$ is a smooth and increasing one-to-one mapping on $(-1,1)$.

On the other hand, the derivative of $h$ is

$$
h^{\prime}(\xi)=\left(\gamma_{r}^{-1}\right)^{\prime}\left(\frac{1+\xi}{2}\right) f^{\prime}\left(2 \gamma_{r}^{-1}\left(\frac{1+\xi}{2}\right)-1\right),
$$

and its asymptotic behaviour near $\xi=1$ and $\xi=-1$ becomes

$$
h^{\prime}(\xi)=O\left((1-\xi)^{(1 / r)-1}\right), \quad h^{\prime}(\xi)=O\left((1+\xi)^{(1 / r)-1}\right),
$$

respectively. Thus $h$ is piecewise smooth on the interval $[-1,1]$ as long as $0<r \leq 1$, which implies the pointwise convergence of the series at both end-points $x= \pm 1$ (that is, $\xi= \pm 1)$. In addition, when $f$ is continuous on $[-1,1]$ and $f(-1)=f(1)$, the uniform convergence of $S^{[r]}$ with $0<r \leq 1$ results from the property of the Fourier series directly. 
THEOREM 5.2. Let $f$ satisfy all of the conditions in Theorem 2.3. Then for all $0<r \leq \eta=\min \left\{\eta_{1}, \eta_{2}\right\}$ we have the following results.

(1) The partial sum $S_{N}^{[r]}(f ; x)$ converges on the interval $[-1,1]$ in the form of $(5.8)$ and (5.9).

(2) If, in addition, $f$ is continuous on $[-1,1]$ then the series $S^{[r]}(f ; x)$ converges uniformly to $f(x)$ on $[-1,1]$.

PROOF. Continuing the proof of Theorem 5.1,

$$
S^{[r]}(f ; x)=S^{\mathrm{F}}(h ; \xi),
$$

in which the asymptotic behaviour of the derivative of $h(\xi)$ becomes

$$
h^{\prime}(\xi)=O\left((1-\xi)^{(1 / r)-1}(1-\xi)^{\left(\eta_{1}-1\right) / r}\right)=O\left((1-\xi)^{\left(\eta_{1} / r\right)-1}\right)
$$

near the right end-point $\xi=1$, and

$$
h^{\prime}(\xi)=O\left((1+\xi)^{\left(\eta_{2} / r\right)-1}\right)
$$

near the left end-point $\xi=-1$. Thus for any $r \leq \eta$ the function $h$ is piecewise smooth on $[-1,1]$. This fact implies the convergence of the Fourier series $S^{\mathrm{F}}(h ; \xi)$ or that of the $\mathrm{SM}-\mathrm{S} S^{[r]}(f ; x)$ as given in (1) and (2).

\section{Numerical examples}

In this section we explore some numerical examples to show the availability of the proposed series. We denote by $E_{N} f$ the $L_{2}$-norm error for the $N$ th partial sum of a series expansion of $f$ on the interval $[-1,1]$. That is,

$$
E_{N} f=\left[\int_{-1}^{1}\left\{f(x)-S_{N}(f ; x)\right\}^{2} d x\right]^{1 / 2}
$$

for an arbitrary partial sum $S_{N}(f ; x)$.

All numerical computations in this section have been performed on a Pentium PC using Mathematica (V.5) software.

EXAMPLE 1. First, we consider a smooth function

$$
f_{1}(x)=1+x(1+x)\left(1-x^{2}\right), \quad-1 \leq x \leq 1 .
$$

Since $f_{1}(-1)=f_{1}(1) \neq 0$, the $N$ th partial sum of the SM-SS in (3.3) should be modified as

$$
S_{\text {sin }}^{[r]}\left(f_{1} ; x\right)=f_{1}(1)+\sum_{n=1}^{\infty} d_{k}^{\prime} \phi_{k}^{[r]}(x),
$$

with

$$
d_{k}^{\prime}=\int_{-1}^{1} w^{[r]}(t) \phi_{k}^{[r]}(t)\left\{f_{1}(t)-f_{1}(1)\right\} d t
$$


TABLE 2. Numerical results of $E_{N} f_{1}$ for the series $S_{\mathrm{cos}, 2 N}^{[r]}\left(f_{1} ; x\right), S_{\mathrm{sin}, 2 N}^{[r]}\left(f_{1} ; x\right), S_{N}^{[r]}\left(f_{1} ; x\right)$ and $S_{N}^{\mathrm{F}}\left(f_{1} ; x\right)$.

\begin{tabular}{|c|c|c|c|c|c|}
\hline \multirow[b]{2}{*}{$r$} & \multirow[b]{2}{*}{$N$} & \multicolumn{3}{|c|}{ Sigmoidal-type series } & \multirow{2}{*}{$\begin{array}{c}\text { Fourier series } \\
\qquad S_{N}^{\mathrm{F}}\left(=S_{N}^{[1]}\right)\end{array}$} \\
\hline & & $S_{\cos , 2 N}^{[r]}$ & $S_{\sin , 2 N}^{[r]}$ & $S_{N}^{[r]}$ & \\
\hline \multirow{5}{*}{1} & 4 & $3.7 \times 10^{-2}$ & $9.4 \times 10^{-3}$ & $2.4 \times 10^{-2}$ & $2.4 \times 10^{-2}$ \\
\hline & 8 & $1.4 \times 10^{-2}$ & $1.8 \times 10^{-3}$ & $9.4 \times 10^{-3}$ & $9.4 \times 10^{-3}$ \\
\hline & 12 & $7.7 \times 10^{-3}$ & $6.5 \times 10^{-4}$ & $5.3 \times 10^{-3}$ & $5.3 \times 10^{-3}$ \\
\hline & 16 & $5.0 \times 10^{-3}$ & $3.2 \times 10^{-4}$ & $3.5 \times 10^{-3}$ & $3.5 \times 10^{-3}$ \\
\hline & 20 & $3.6 \times 10^{-3}$ & $1.8 \times 10^{-4}$ & $2.5 \times 10^{-3}$ & $2.5 \times 10^{-3}$ \\
\hline \multirow{5}{*}{$\frac{1}{2}$} & 4 & $8.8 \times 10^{-4}$ & $2.6 \times 10^{-3}$ & $9.8 \times 10^{-4}$ & - \\
\hline & 8 & $2.6 \times 10^{-5}$ & $2.2 \times 10^{-4}$ & $1.4 \times 10^{-4}$ & - \\
\hline & 12 & $5.5 \times 10^{-6}$ & $7.2 \times 10^{-5}$ & $4.8 \times 10^{-5}$ & - \\
\hline & 16 & $1.8 \times 10^{-6}$ & $3.2 \times 10^{-5}$ & $2.1 \times 10^{-5}$ & - \\
\hline & 20 & $7.7 \times 10^{-7}$ & $1.7 \times 10^{-5}$ & $1.1 \times 10^{-5}$ & - \\
\hline \multirow{5}{*}{$\frac{1}{4}$} & 4 & $7.6 \times 10^{-2}$ & $7.8 \times 10^{-2}$ & $6.2 \times 10^{-2}$ & - \\
\hline & 8 & $2.0 \times 10^{-3}$ & $2.8 \times 10^{-3}$ & $1.8 \times 10^{-3}$ & - \\
\hline & 12 & $3.2 \times 10^{-5}$ & $4.9 \times 10^{-5}$ & $3.0 \times 10^{-5}$ & - \\
\hline & 16 & $3.8 \times 10^{-7}$ & $6.2 \times 10^{-7}$ & $3.6 \times 10^{-7}$ & - \\
\hline & 20 & $3.0 \times 10^{-9}$ & $7.0 \times 10^{-9}$ & $6.9 \times 10^{-9}$ & - \\
\hline
\end{tabular}

Since $f_{1}(x)$ is continuous as well as piecewise smooth on the interval $[-1,1]$, Theorems 2.2, 3.2 and 5.1 imply that all of the SM-CS $S_{\text {cos }}^{[r]}\left(f_{1} ; x\right)$, the SM-SS $S_{\sin }^{[r]}\left(f_{1} ; x\right)$ and the SM-S $S^{[r]}\left(f_{1} ; x\right)$ converge uniformly to the original function $f_{1}(x)$ on $[-1,1]$ for any $0<r \leq 1$.

Table 2 includes numerical results of the partial sums $S_{\cos , 2 N}^{[r]}\left(f_{1} ; x\right), S_{\sin , 2 N}^{[r]}\left(f_{1} ; x\right)$ and $S_{N}^{[r]}\left(f_{1} ; x\right), r=1,1 / 2,1 / 4$, of the sigmoidal-type series developed in this work. One can see that the sigmoidal-type partial sums with $r<1$ greatly improve the $L_{2}$ norm errors of the traditional Fourier series, except for the case of $N=4$ with $r=1 / 4$. When $r=1$ the errors of $S_{\mathrm{cos}, 2 \mathrm{~N}}^{[r]}\left(f_{1} ; x\right)$ for $N=4(4) 20$ are all greater than those of the Fourier series $S_{N}^{\mathrm{F}}\left(f_{1} ; x\right)=S_{N}^{[1]}\left(f_{1} ; x\right)$.

Figure 2 shows the outstanding uniform convergence of the partial sum $S_{N}^{[1 / 2]}\left(f_{1} ; x\right)$ compared with the Fourier partial sum $S_{N}^{\mathrm{F}}\left(f_{1} ; x\right)$ for small values of $N$. In addition, the $L_{2}$-norm errors of $S_{N}^{[r]}\left(f_{1} ; x\right)$ with respect to the order $1 / 8 \leq r \leq 1$ (that is, $1 / r=1,1.5,2, \ldots, 8$ ) are illustrated in Figure 3. Therein the horizontal lines indicate the errors of $S_{N}^{\mathrm{F}}\left(f_{1} ; x\right)$. We can observe that the optimal errors occur near $r=1 / 4$.

EXAMPLE 2. We consider a 2-periodic continuous function

$$
f_{2}(x)=e^{x} \sqrt{1-x^{2}}, \quad-1 \leq x \leq 1,
$$

with $f_{2}(x+2)=f_{2}(x)$ for all $x \in \mathbb{R}$. 

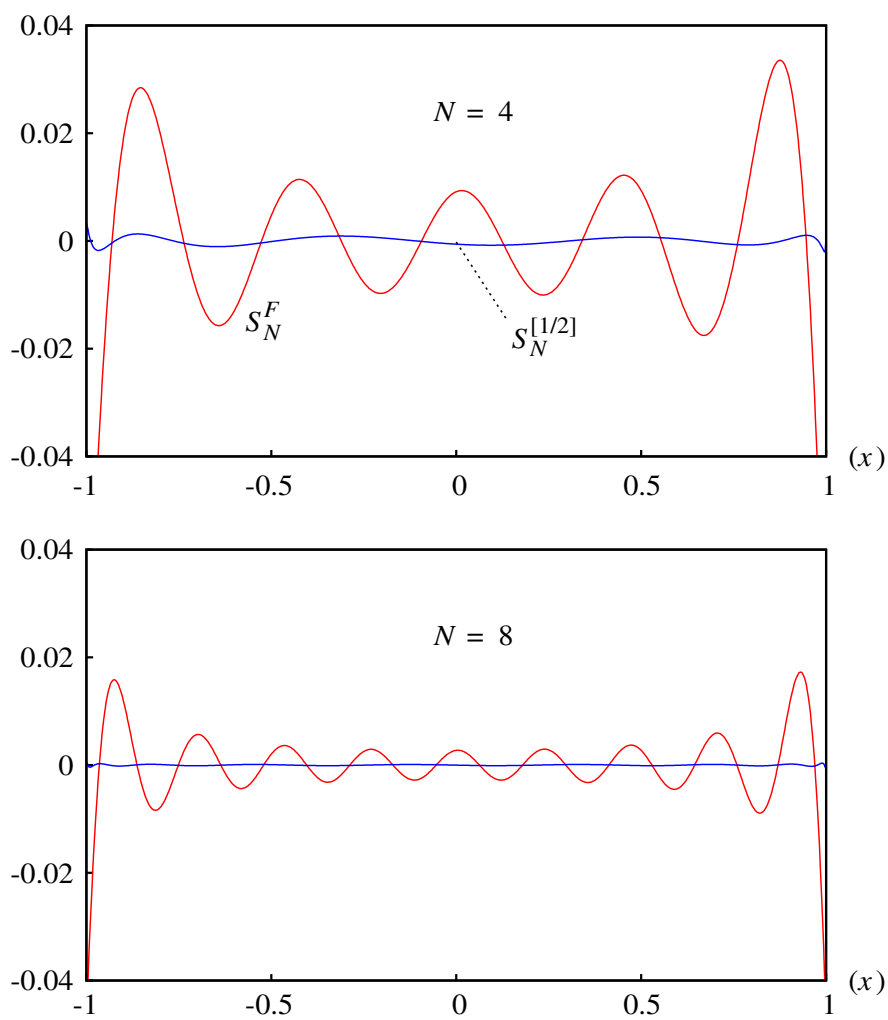

FIGURE 2. Errors $f_{1}(x)-S_{N}^{\mathrm{F}}\left(f_{1} ; x\right)$ and $f_{1}(x)-S_{N}^{[1 / 2]}\left(f_{1} ; x\right)$ for $N=4$ and 8 .

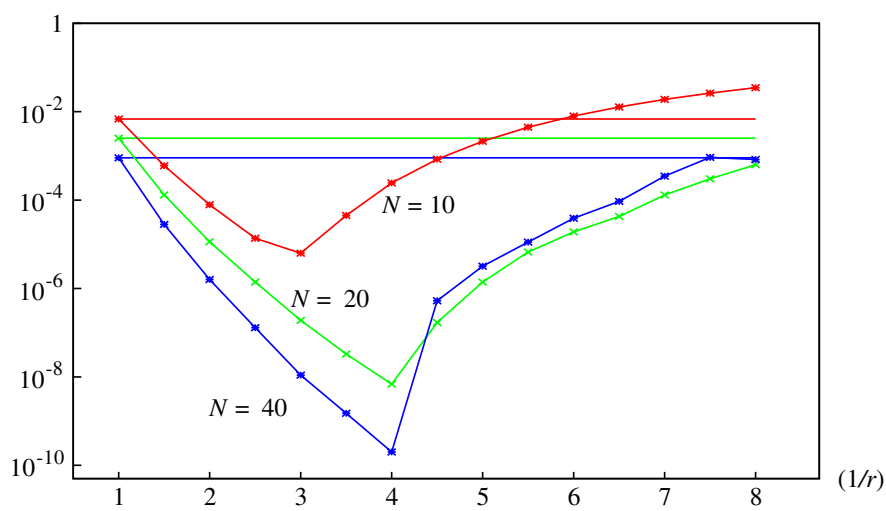

FIGURE 3. $L_{2}$-norm errors $E_{N} f_{1}, N=10,20,40$, for the partial sums of the sigmoidal series, $S_{N}^{[r]}\left(f_{1} ; x\right)$ for $1 \leq 1 / r \leq 8$. 
TABLE 3. Numerical results of $E_{N} f_{2}$ for the series $S_{\mathrm{cos}, 2 N}^{[r]}\left(f_{2} ; x\right), S_{\sin , 2 N}^{[r]}\left(f_{2} ; x\right), S_{N}^{[r]}\left(f_{2} ; x\right)$ and $S_{N}^{\mathrm{F}}\left(f_{2} ; x\right)$.

\begin{tabular}{cccccc}
\hline & & \multicolumn{3}{c}{ Sigmoidal-type series } & Fourier series \\
\cline { 3 - 5 }$r$ & $N$ & $S_{\text {cos }, 2 N}^{[r]}$ & $S_{\text {sin,2N }}^{[r]}$ & $S_{N}^{[r]}$ & $S_{N}^{\mathrm{F}}\left(=S_{N}^{[1]}\right)$ \\
\hline & 4 & $1.4 \times 10^{-2}$ & $1.2 \times 10^{-3}$ & $1.0 \times 10^{-2}$ & $9.6 \times 10^{-2}$ \\
& 8 & $4.1 \times 10^{-3}$ & $1.6 \times 10^{-4}$ & $3.1 \times 10^{-3}$ & $5.1 \times 10^{-2}$ \\
$\frac{1}{2}$ & 12 & $1.9 \times 10^{-3}$ & $5.0 \times 10^{-4}$ & $1.5 \times 10^{-3}$ & $3.5 \times 10^{-2}$ \\
& 16 & $1.1 \times 10^{-3}$ & $2.2 \times 10^{-5}$ & $8.8 \times 10^{-4}$ & $2.6 \times 10^{-2}$ \\
& 20 & $7.5 \times 10^{-4}$ & $1.2 \times 10^{-5}$ & $5.8 \times 10^{-4}$ & $2.1 \times 10^{-2}$ \\
& & & & & \\
& 4 & $2.3 \times 10^{-2}$ & $2.2 \times 10^{-3}$ & $1.6 \times 10^{-2}$ & - \\
$\frac{1}{4}$ & 8 & $2.3 \times 10^{-4}$ & $4.6 \times 10^{-4}$ & $2.5 \times 10^{-4}$ & - \\
& 12 & $3.7 \times 10^{-6}$ & $3.9 \times 10^{-5}$ & $2.3 \times 10^{-5}$ & - \\
& 16 & $8.7 \times 10^{-7}$ & $1.6 \times 10^{-5}$ & $9.0 \times 10^{-6}$ & - \\
& 20 & $3.7 \times 10^{-7}$ & $8.2 \times 10^{-6}$ & $4.7 \times 10^{-6}$ & - \\
& 4 & $1.1 \times 10^{-1}$ & $7.8 \times 10^{-2}$ & $7.8 \times 10^{-2}$ & - \\
& 8 & $5.7 \times 10^{-3}$ & $6.6 \times 10^{-3}$ & $5.0 \times 10^{-3}$ & - \\
$\frac{1}{6}$ & 12 & $3.0 \times 10^{-4}$ & $4.2 \times 10^{-4}$ & $2.9 \times 10^{-4}$ & - \\
& 16 & $1.9 \times 10^{-5}$ & $2.1 \times 10^{-5}$ & $1.6 \times 10^{-5}$ & - \\
& 20 & $1.1 \times 10^{-6}$ & $9.0 \times 10^{-7}$ & $8.8 \times 10^{-7}$ & - \\
\hline & & & & & \\
\hline
\end{tabular}

The function $f_{2}(x)$ is not piecewise smooth on $[-1,1]$ because its derivative is unbounded at the end-points $x= \pm 1$. Thereby, even though its Fourier series converges to $f_{2}(x)$ in $L_{2}$-norm, it cannot guarantee pointwise convergence nor uniform convergence. In contrast, Theorems $2.3,3.3$ and 5.2 imply that all the sigmoidal-type series $S_{\mathrm{cos}}^{[r]}\left(f_{2} ; x\right), S_{\sin }^{[r]}\left(f_{2} ; x\right)$ and $S^{[r]}\left(f_{2} ; x\right)$ converge uniformly to $f_{2}$ as long as $0<r \leq \eta=1 / 2$.

Table 3 shows that the $L_{2}$-norm errors $E_{N} f_{2}$ of $S_{\cos , 2 N}^{[r]}\left(f_{2} ; x\right), S_{\sin , 2 N}^{[r]}\left(f_{2} ; x\right)$ and $S_{N}^{[r]}\left(f_{2} ; x\right)$ with $r=1 / 2,1 / 4,1 / 6$ become much better than those of $S_{N}^{\mathrm{F}}\left(f_{2} ; x\right)$ as the value of $N$ grows larger.

Figure 4 compares the differences $f_{2}-S_{N}^{\mathrm{F}}\left(f_{2} ; x\right)$ and $f_{2}-S_{N}^{[1 / 4]}\left(f_{2} ; x\right)$ for $N=$ 4,8 , which implies uniform convergence of the present SM-S in contrast with the Fourier series $S_{N}^{\mathrm{F}}\left(f_{2} ; x\right)$. For each $N=10,20,30$, numerical results of the errors $E_{N} f_{2}$ for $S_{N}^{[r]}\left(f_{2} ; x\right)$ with respect to various $r$ are shown in Figure 5. It is seen that for all $r$ over the interval $1<1 / r \leq 8$ the partial $\operatorname{sum} S_{N}^{[r]}\left(f_{2} ; x\right)$ is very efficient compared with the Fourier partial sum $S_{N}^{\mathrm{F}}\left(f_{2} ; x\right)$.

On the other hand, concerning the 2-periodicity of the given function $f_{2}(x)$ on the real line $\mathbb{R}$, we can take either the ESM-S $\widetilde{S}^{[r]}\left(f_{2} ; x\right)$ in (5.1) or the ESM-SS $\widetilde{S}_{\sin }^{*[r]}\left(f_{2} ; x\right)$ in (5.5). For instance, Figure 6 shows the approximations to $f_{2}(x)$ by the partial sums $S_{N}^{\mathrm{F}}\left(f_{2} ; x\right)$ and $\widetilde{S}_{N}^{[1 / 2]}\left(f_{2} ; x\right)$ of the Fourier series $S^{\mathrm{F}}\left(f_{2} ; x\right)$ and the 

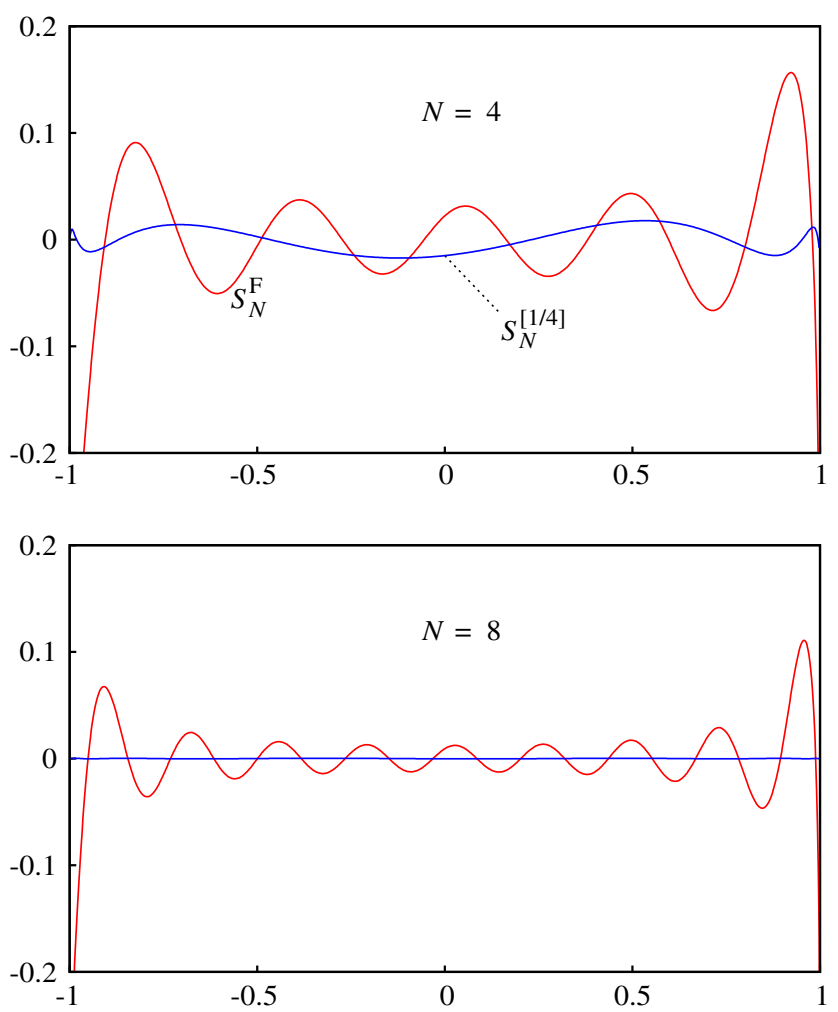

$(x)$

FiguRE 4. Errors $f_{2}(x)-S_{N}^{\mathrm{F}}\left(f_{2} ; x\right)$ and $f_{2}(x)-S_{N}^{[1 / 4]}\left(f_{2} ; x\right)$ for $N=4$ and 8.

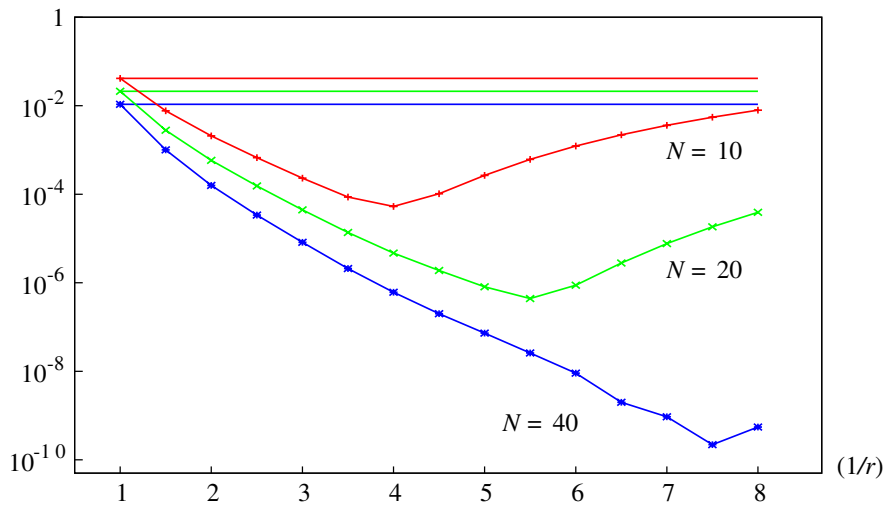

FIGURE 5 . $L_{2}$-norm errors $E_{N} f_{2}, N=10,20,40$, for the partial sums of the sigmoidal series, $S_{N}^{[r]}\left(f_{2} ; x\right)$ for $1 \leq 1 / r \leq 8$. 

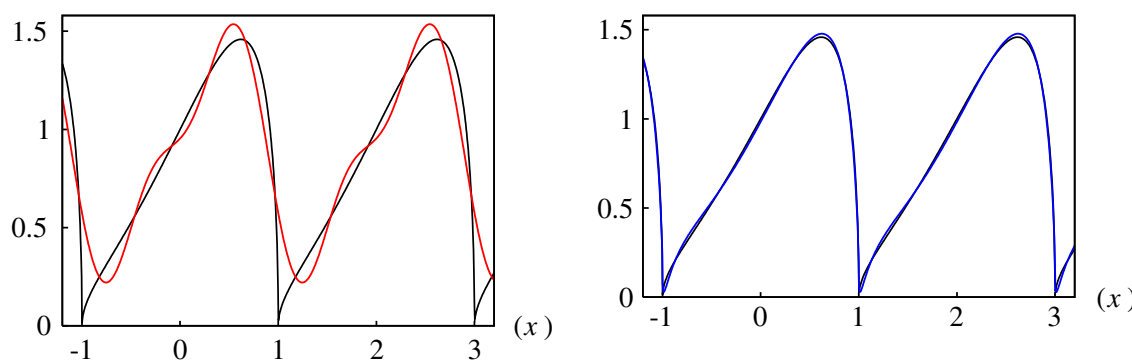

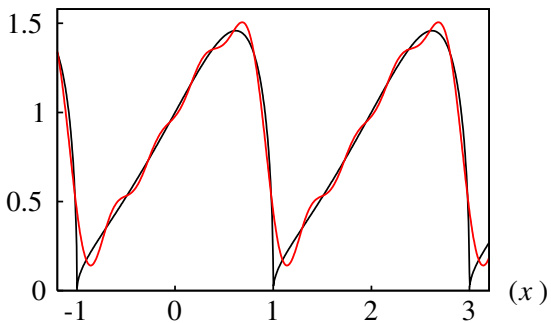

(a)

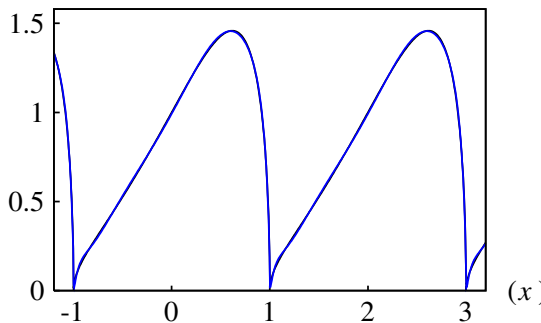

(b)

FIGURE 6. The 2-periodic approximation to the function $f_{2}(x)$ by the Fourier series $S_{N}^{F}\left(f_{2} ; x\right)$ (in (a)) and the ESM-S $\widetilde{S}_{N}^{[1 / 2]}\left(f_{2} ; x\right)$ (in (b)), for $N=2$ (upper row) and $N=4$ (lower row).

ESM-S $\widetilde{S}^{[1 / 2]}\left(f_{2} ; x\right)$, respectively. Though the number of the sum is very small, such as $N=2$ or $4, \widetilde{S}_{N}^{[1 / 2]}\left(f_{2} ; x\right)$ seems to be decidedly superior to $S_{N}^{\mathrm{F}}\left(f_{2} ; x\right)$. By further numerical experiment one can find that the partial sum $\widetilde{S}_{\sin , N}^{*[r]}\left(f_{2} ; x\right)$ of the ESM-SS $\widetilde{S}_{\sin }^{*[r]}\left(f_{2} ; x\right)$ with $r \leq 1 / 2$ also gives similar results to those of $\widetilde{S}_{N}^{[r]}\left(f_{2} ; x\right)$.

\section{References}

[1] U. J. Choi, S. W. Kim and B. I. Yun, "Improvement of the asymptotic behavior of the EulerMaclaurin formula for Cauchy principal value and Hadamard finite-part integrals", Int. J. Numer. Meth. Engng. 61 (2004) 496-513.

[2] D. Elliott, "The Euler-Maclaurin formula revisited", J. Austral. Math. Soc. Ser. B 40(E) (1998) E27-E76.

[3] D. Elliott, "Sigmoidal transformations and the trapezoidal rule", J. Austral. Math. Soc. Ser. B 40(E) (1998) E77-E137.

[4] D. Elliott and E. Venturino, "Sigmoidal transformations and the Euler-Maclaurin expansion for evaluating certain Hadamard finite-part integrals", Numer. Math. 77 (1997) 453-465.

[5] P. R. Johnston, "Application of sigmoidal transformations to weakly singular and near singular boundary element integrals", Int. J. Numer. Meth. Engng. 45 (1999) 1333-1348.

[6] S. Prössdorf and A. Rathsfeld, "On an integral equation of the first kind arising from a cruciform crack problem", in Integral equations and inverse problems (eds. V. Petkov and R. Lazarov), (Longman, Harlow, 1991) 210-219. 
[7] A. Sidi, "A new variable transformation for numerical integration", in Numerical integration IV, Volume 112 of International Series of Numerical Mathematics (eds. H. Brass and G. Hämmerlin), (Birkhäuser, Basel, 1993) 359-373.

[8] B. I. Yun, "Sigmoidal cosine series on the interval", ANZIAM J. 47 (2006) 451-475.

[9] B. I. Yun, "A non-linear co-ordinate transformation for accurate numerical evaluation of weakly singular integrals", Comm. Numer. Methods Engrg. 20 (2004) 401-417.

[10] B. I. Yun, "An extended sigmoidal transformation technique for evaluating weakly singular integrals without splitting the integration interval", SIAM J. Sci. Comput. 25 (2003) 284-301.

[11] B. I. Yun and P. Kim, "A new sigmoidal transformation for weakly singular integrals in the boundary element method", SIAM J. Sci. Comput. 24 (2003) 1203-1217. 